\title{
On the initial development of shear layers in partially vegetated channels
}

\author{
Adriano Coutinho de LIMA ${ }^{1}$ and Norihiro IZUMI ${ }^{2}$ \\ ${ }^{1}$ Member of JSCE, Ph.D., Assistant Professor, Division of Field Engineering for Environment, Graduate School of \\ Engineering, Hokkaido University (Kita 13, Nishi 8, Kita-ku, Sapporo, Hokkaido, 060-8628, Japan) \\ ${ }^{2}$ Member of JSCE, Ph.D., Professor, Division of Field Engineering for Environment, Graduate School of Engineer- \\ ing, Hokkaido University (Kita 13, Nishi 8, Kita-ku, Sapporo, Hokkaido, 060-8628, Japan)
}

\begin{abstract}
Linear stability analysis of flows in open-channels with vegetation at one of their sides is performed. The lateral gradient of the streamwise velocity induced by the drag difference between the vegetated zone and the adjacent non-vegetated zone may result in flow instability in the shear layer around the edge of the vegetated zone, causing the generation of discrete horizontal vortices.

We assume that the base state flow field before the occurrence of instability is characterized by a sub-depth-scale turbulence which is mainly generated by bottom friction. By introducing perturbations to the flow depth as well as the streamwise and transverse velocities in the base state, the conditions required for perturbations grow in time were studied over a wide range of hydraulic parameters. The theory is validated by means of its application to experimental data.
\end{abstract}

Key Words : Linear stability analysis, shear flow, flow instability, kinematic eddy viscosity.

\section{INTRODUCTION}

Vegetation located at one side of a channel produces velocity inflection and transverse shear, which may lead to flow instability and the generation of large-scale horizontal vortices. These vortices have a strong influence on the velocity distribution and on the amount of discharge conveyed by a channel without overflow, and enhance the lateral exchange of mass and momentum between the vegetated zone and the open channel. Similar transverse shear flows are also observed for example in composite channels, ${ }^{1,2)}$ in inflows at channel junctions, ${ }^{3,4)}$ or in channels where the bottom roughness varies laterally. ${ }^{2)}$ Tamai et al. ${ }^{1)}$ concluded that the predominant factor in the generation of the large eddies observed in a set of compound channels was the shear layer in the lateral velocity profile. Chu et al. ${ }^{2)}$ pointed that the stability of transverse shear flows is dependent not only on the magnitude of transverse shear, but also on the degree of bed-friction influence. Additionally, Kolyshkin and Ghidaoui ${ }^{5)}$ highlighted the influence of gravity on the stability of transverse shear flows.
Stability characteristics of open channel flows with transverse shear have been studied by means of linear stability analysis by a number of authors. The linear stability analysis can successfully explain the effects of various hydraulics parameters in the stability of the flow. In Refs. 2, 6, 7, and 8, the analysis was performed for the inviscid case, therefore reducing the perturbation equations to modified Rayleigh equations, while in Refs. 4 and 8 the viscous terms were kept in the perturbed equations.

A temporal linear stability analysis of flow in an open-channel partially covered with vegetation is performed in this study. By not employing the rigid-lid and the inviscid flow assumptions, we can study the effect of the Froude number and the kinematic eddy viscosity, respectively, on the growth rate of perturbations. We employ the St. Venant shallow water equations with the Reynolds stress expressed by a kinematic eddy viscosity representing turbulence with a length scale smaller than the flow depth. Differently from Refs. 4 and 8, this eddy viscosity is estimated for the flow unaffected by the large-scale horizontal vortices; therefore, it represents only the turbulence generated by the bottom 
friction. We study how various hydraulic parameters affect the time development of the perturbations.

\section{GOVERNING EQUATIONS OF FLUID MOTION}

In this study we focus on the initial development of horizontal vortices generated in the shallow flow in a wide rectangular open-channel partially covered with emergent rigid vegetation, as depicted in Fig. 1. We employ the formulation described in Lima and Izumi, ${ }^{9}$ which on the other hand focused on the fully developed state of the horizontal vortices. The vegetation is modeled by an array of regularly spaced cylinders with a uniform diameter installed only on one side of the channel. The horizontal length scale of the vortices is commonly large compared with the scale of the flow depth. The generation of such thin vortices can be described by the depth-averaged shallow water formulation. The momentum equations in the streamwise and transverse directions $(x$ and $y$ ) and the continuity equation are

$$
\begin{aligned}
& \frac{\partial U}{\partial t}+U \frac{\partial U}{\partial x}+V \frac{\partial U}{\partial y}=-F^{-2} \frac{\partial H}{\partial x}+\beta\left(1-\frac{T_{b x}+D_{x}}{H}\right) \\
&+\epsilon\left(\frac{\partial^{2} U}{\partial x^{2}}+\frac{\partial^{2} U}{\partial y^{2}}\right), \\
& \frac{\partial V}{\partial t}+U \frac{\partial V}{\partial x}+V \frac{\partial V}{\partial y}=-F^{-2} \frac{\partial H}{\partial y}-\beta \frac{T_{b y}+D_{y}}{H}+\epsilon\left(\frac{\partial^{2} V}{\partial x^{2}}+\frac{\partial^{2} V}{\partial y^{2}}\right), \\
& \frac{\partial H}{\partial t}+\frac{\partial U H}{\partial x}+\frac{\partial V H}{\partial y}=0,
\end{aligned}
$$

where $t$ is time, $x$ is the streamwise coordinate, $y$ is the lateral coordinate, the origin of which is taken at the interface between the vegetated and non-vegetated zones, $U$ and $V$ are the $x$ and $y$ components of the flow velocity respectively, $H$ is the flow depth, $T_{b x}$ and $T_{b y}$ are the $x$ and $y$ components of the bed shear stress respectively and $D_{x}$ and $D_{y}$ are the $x$ and $y$ components of the drag force due to vegetation respectively,

$\left(T_{b x}, T_{b y}\right)=\left(U^{2}+V^{2}\right)^{1 / 2}(U, V)$,

$$
\left(D_{x}, D_{y}\right)=\left\{\begin{array}{c}
\alpha\left(U^{2}+V^{2}\right)^{1 / 2} H(U, V) \text { if }-B v \leq y \leq 0 \\
0 \text { if } 0 \leq y \leq 1 .
\end{array}\right.
$$

The variables in (1)-(5) have been rendered dimensionless in the form

$$
\begin{aligned}
(\widetilde{U}, \tilde{V}) & =\widetilde{U}_{\infty}(U, V), \quad \widetilde{H}=\widetilde{H}_{\infty} H, \\
(\tilde{x}, \tilde{y}) & =\tilde{B}(x, y), \quad \tilde{t}=\frac{\widetilde{B}}{\widetilde{U}_{\infty}} t,
\end{aligned}
$$

where $\widetilde{U}_{\infty}$ and $\widetilde{H}_{\infty}$ are the velocity and flow depth in the region sufficiently far from the vegetated zone, and $\tilde{B}$ is the width of the non-vegetated zone. The tilde is used to indicate dimensional variables. The normalized transverse coordinate $y$ extends from $-B_{v}$ to 1 , where $B_{v}$ is the normalized width of the vegetated zone.

Equations (1)-(5) contain the non-dimensional parameters $\beta, \epsilon, F$ and $\alpha$ which are expressed as $\beta=\frac{C_{f} \tilde{B}}{\widetilde{H}_{\infty}}, \epsilon=\frac{\widetilde{v}_{T}}{\widetilde{U}_{\infty} \tilde{B}}, F=\frac{\widetilde{U}_{\infty}}{\sqrt{\tilde{g} \widetilde{H}_{\infty}}}, \alpha=\frac{C_{D} \tilde{a} \widetilde{H}_{\infty}}{2 C_{f}}$.
The parameter $\beta$ expresses the relative importance of the bed shear effect and is dependent on the bottom friction coefficient $C_{f}$, which is assumed as constant for simplicity. The parameter $\epsilon$ measures the relative importance of the sub-depth kinematic eddy viscosity $\tilde{v}_{T}, F$ is the Froude number sufficiently far from the vegetation and $\alpha$ measures the relative importance of the shear instability produced by the vegetation drag, expressed by the coefficient $C_{D}$, and density, expressed by $\tilde{a}=\tilde{d} /\left(\tilde{l}_{x} \tilde{l}_{y}\right)$, where $\tilde{d}$ is the diameter of the cylinders and $\tilde{l}_{x}$ and $\tilde{l}_{y}$ are the distances between two adjacent cylinders in the $x$ and $y$ directions respectively.

It is assumed that the channel is sufficiently wide so that the shear layer does not reach the side walls. As a consequence, the transverse velocity $V$ vanishes, and the streamwise velocity $U$ asymptotically approaches to constant slip velocities at the side walls:

$$
\frac{\partial U}{\partial y}=0 \text { at } y=-B_{v}, 1, \quad V=0 \text { at } y=-B_{v}, 1 .
$$

Right at the boundary between the non-vegetated and vegetated zones, the velocities, flow depth and lateral shear stresses are continuous,

$$
\begin{aligned}
\lim _{\mathrm{y} \rightarrow+0}\left(U, V, H, \frac{\partial U}{\partial x}, \frac{\partial U}{\partial y}+\frac{\partial V}{\partial x}, \frac{\partial V}{\partial y}\right) \\
=\lim _{\mathrm{y} \rightarrow-0}\left(U, V, H, \frac{\partial U}{\partial x}, \frac{\partial U}{\partial y}+\frac{\partial V}{\partial x}, \frac{\partial V}{\partial y}\right) .
\end{aligned}
$$

\section{(1) Sub-depth kinematic eddy viscosity}

We assume that, in the base state before instability occurs, the flow is already affected by turbulence the length scale of which is smaller than the flow depth (sub-depth scale turbulence). Where there is no influence of vegetation, the kinematic eddy viscosity $\tilde{v}_{T}$ should correspond to the sub-depth scale turbulence generated by the bottom friction. We employ a logarithmic velocity distribution as a sub-depth scale turbulent velocity distribution due to the bottom friction. The kinematic eddy viscosity then takes a parabolic form which is depth-averaged from the bottom to the water surface yielding

$$
\widetilde{v}_{T}=\frac{1}{6} \kappa \widetilde{U}_{f} \widetilde{H}_{\infty}=0.067 \widetilde{U}_{f} \widetilde{H}_{\infty},
$$

where $\widetilde{U}_{f}$ is the friction velocity in the region sufficiently far from the vegetated zone and $\kappa$ is the
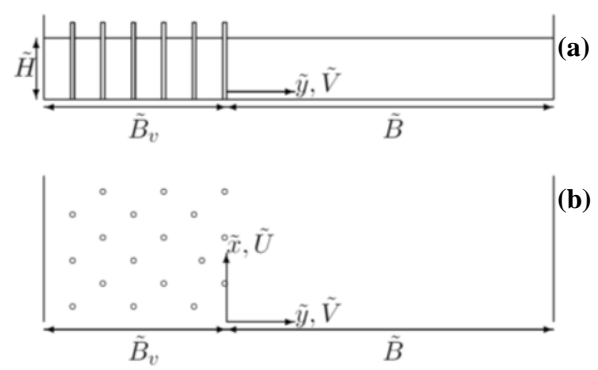

Fig.1 Sketch of the partially vegetated channel: (a) cross-section; (b) plan view. 
Karman constant $(=0.4)$. We assume that the sub-depth scale turbulence is rather isotropic. Therefore, (10) is expected to describe the Reynolds stresses in the streamwise and lateral directions at a sufficient distance from the vegetated zone. In addition, although the velocity and the shear velocity are reduced in the shear layer and in the vegetated zone, and the length scale of the sub-depth scale vortices may be affected by a typical length scale of vegetation such as the vegetation spacing, $\tilde{v}_{T}$ can be represented by (10) even in the shear layer and in the vegetated zone. This may be attributed to the fact that the sum of the resistant forces (the bed shear stress, the Reynolds stress and the vegetation drag force) remains constant regardless of the reduction in the bed shear stress in the shear layer and the vegetated zone, as suggested by the experimental results of Ikeda et $\left.a l .{ }^{6}\right)$ Furthermore, since the flow depth and the spacing of vegetation in Ikeda et al.' $\mathrm{s}^{6}$ experiments are both in the same range, the kinematic eddy viscosity in the vegetated zone may not be strongly affected by vegetation.

Fischer et al. ${ }^{10)}$ determined the transverse mixing coefficient $\tilde{e}_{T} \cong 0.15 \widetilde{U}_{f} \widetilde{H}_{\infty}$ from an approximate average of experimental results in straight rectangular channels from various studies. Based on Fischer et al., ${ }^{10)}$ Chen and Jirka ${ }^{11)}$ employed the transverse eddy viscosity $\widetilde{v}_{T}=0.2 \widetilde{U}_{f} \widetilde{H}_{\infty}$, followed by Ghidaoui and Kolyshkin ${ }^{8)}$ and Prooijen and Uijttewaal, ${ }^{4)}$ the latter employing $\widetilde{v}_{T}=0.15 \widetilde{U}_{f} \widetilde{H}_{\infty}$. Although the eddy viscosity of Chen and Jirka ${ }^{11)}$ is based on the small-scale turbulence, it is estimated for the flow where lateral large-scale transverse motions occur. On the other side, the eddy viscosity in (10) is estimated for the flow undisturbed by large-scale transverse motions, resulting in a value smaller than that of Chen and Jirka. ${ }^{11)}$ By employing (10) in the formulation, we can set a base flow which consists in the flow completely undisturbed by the large-scale lateral motions, differently from previous studies, e.g., Refs. 2, 4, 5, 6, 7, where the average flow was taken as the base flow field.

\section{LINEAR STABILITY ANALYSIS}

A disturbance undulating in the streamwise direction is introduced to the base state flow undisturbed by the horizontal vortices. The streamwise and lateral velocities $U$ and $V$, and the flow depth $H$ are then expanded in the form

$$
\begin{gathered}
U(x, y, t)=U_{0}(y)+A U_{1}(y) e^{i(k x-\omega t)} \\
V(x, y, t)=A V_{1}(y) e^{i(k x-\omega t)} \\
H(x, y, t)=1+A H_{1}(y) e^{i(k x-\omega t)}
\end{gathered}
$$

where $A, k$ and $\omega$ are the amplitude (assumed to be small such that $A^{2} \rightarrow 0$ ), wavenumber and angular frequency of the perturbation respectively. In the scheme of temporal linear stability analysis, employed herein, $k$ is real while $\omega$ is complex as $\omega=\omega_{r}+i \Omega$, where $\omega_{r}$ is the real angular frequency and $\Omega$ is the growth rate of perturbation.

Under the base state normal flow condition $V$ vanishes, $H$ is constant $(=1)$ and $U$ is a function of only the transverse coordinate $(y)$ denoted by $U_{0}$ and analytically determined as

$$
U_{0}=\left\{\begin{array}{c}
3 \tanh ^{2}\left[\left(\frac{\beta}{2 \epsilon}\right)^{1 / 2} y+\tanh ^{-1}\left(\frac{\psi+2}{3}\right)^{1 / 2}\right]-2 \\
\text { if } 0 \leq y \leq 1 ; \\
3 \phi \operatorname{coth}^{2}\left[-\left(\frac{\beta}{2 \epsilon \phi}\right)^{1 / 2} y+\operatorname{coth}^{-1}\left(\frac{\psi+2 \phi}{3 \phi}\right)^{1 / 2}\right]-2 \phi \\
\text { if }-B v \leq y \leq 0,
\end{array}\right.
$$

where $\phi$ is the ratio of the undisturbed velocities in the vegetated $\left(\widetilde{U}_{-\infty}\right)$ and non-vegetated $\left(\widetilde{U}_{\infty}\right)$ zones,

$$
\phi=\frac{\widetilde{U}_{-\infty}}{\widetilde{U}_{\infty}}=\frac{1}{(1+\alpha)^{1 / 2}},
$$

and $\psi$ is the base flow velocity at $y=0$, related to $\phi$ as

$$
\psi=\left(\frac{2 \phi^{2}}{1+\phi}\right)^{1 / 3} \text {. }
$$

The base state velocity $U_{0}$ and measurements of the streamwise velocity $U$ at the developed state of the perturbations are presented in Fig. 2 for run 1 of the experiments of Ikeda et al. ${ }^{12)}$ (see Table 1). The shear layer of $U_{0}$ is narrower than the shear layer of the measurements because the base state corresponds to the flow before large-scale perturbations take place, while the measurements correspond to a developed state where the perturbations have evolved to a nonlinear instability regime.

The equations at the order of $A$ obtained from the introduction of the perturbation expansions (11)-(13) into (1)-(3) (as well as into the boundary and matching conditions (8a,b)-(9)) do not admit analytical solutions. We then employ a spectral collocation method with the Chebyshev polynomials. After some algebraic manipulation, the resulting system of equations takes the functional form $\omega=\omega\left(\beta, \epsilon, \alpha, B_{v}, F, k\right)$.

\section{RESULTS}

\section{(1) Stability domains}

The contours of $\Omega$ on the plane as functions of the parameters $\beta, \epsilon, \alpha, F$ and $k$ are shown in

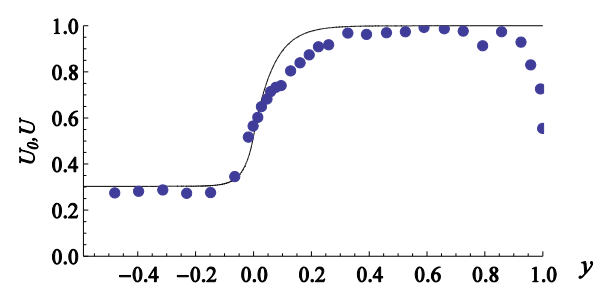

Fig. 2 Base state velocity $U_{0}$ (continuous line) and measured streamwise velocity $U$ at the developed state of perturbations for run 1 of Ikeda et al. ${ }^{12)}$ 
Figs. 3-6. In this analysis, we assume that $B_{v}$ is large enough so that $U_{0}$ is almost constant at $y=-B_{v}$, implying that $\Omega$ is almost independent of $B_{v}$. When $\Omega$ is positive, the perturbations grow as time progresses, while when $\Omega$ is negative the perturbations decay to vanish. In the figures, $\Omega$ typically becomes negative in the range of sufficiently small and large values of wavenumber $k$, and takes a maximum value between them. It follows that $\Omega$ as a function of $k$ commonly possesses a characteristic wavenumber $k_{m}$ associated with the maximum growth rate $\Omega_{m}$, implying the selection of a preferential wavelength at the linear level.

The dependence of $\Omega$ on $\beta$ is studied in Fig. 3. The effect of $\beta$ on $\Omega$ is dual: in the range of large $\beta, \Omega$ is reduced because the effect of the bottom friction inhibits the effect of the lateral velocity gradient and attenuates the large-scale turbulence, as already shown by Chu et al. ${ }^{2)}$ and White and Nepf. ${ }^{7)}$ On the other side, in the range of small $\beta, \Omega$ is also reduced because of the reduced small-scale turbulences. Accordingly, in Fig. 3, as $\beta$ increases or decreases from approximately $10^{-1}$, where $\Omega$ is maximized, the flow becomes less stable, and in the range of $\beta \gtrsim 0.5$, the region of positive $\Omega$ completely disappears. Although the present shallow water formulation may not be valid in the range of $\beta \lesssim 5 \times 10^{-3}$, the decrease in the growth rate is expected to occur in this range. In the range of sufficiently small $\beta$, the shear layer may be affected by the zero disturbance boundary condition of the walls. ${ }^{5)}$ The range of $k$ for positive $\Omega$ and the characteristic wavenumber $k_{m}$ increase with $\beta$ because the wavenumber $k$ is normalized by the width of the non-vegetated zone $\tilde{B}$.

The effect of the parameter $\epsilon$ on $\Omega$ is studied in Fig. 4, where it is found that the flow becomes stable when $\epsilon \gtrsim 4 \times 10^{-3}$ under the conditions of the figure. In this range, the dissipation of energy caused by the small-scale turbulences will be sufficiently large to suppress the effect of the transverse mixing. On the other side, as $\epsilon$ decreases, $\Omega$ increases. In the range of very small $\epsilon$, the flow approaches to the inviscid case. The characteristic wavenumber $k_{m}$ increases with the decrease of $\epsilon$ because of the normalization of the wavenumber $k$. Because $\tilde{v}_{T}$ is derived taking into consideration the small-scale turbulences generated by the bottom friction before instability takes place, $\epsilon$ does not contain the effect of the large-scale turbulences. In case we had employed the kinematic eddy viscosity as in Chen and Jirka, ${ }^{11)}$ the growth rate of perturbations would have been underestimated, following that a larger eddy viscosity would correspond to a smaller growth rate.

The dependence of $\Omega$ on the vegetation drag parameter $\alpha$ is studied in Fig. 5, where it is found that, in the range of small $\alpha, \Omega$ is negative and the flow is stable as already pointed out by Chu et al. ${ }^{2)}$ and White and Nepf. ${ }^{7)}$ As $\alpha$ increases, $\Omega$ increases with a slight increase in $k_{m}$, which peaks around the point $(\alpha ; k) \approx\left(10^{2}, 6\right)$. In the range of $\alpha \gtrsim 10^{2}, \Omega$ decreases with slight decreases in $k_{m}$, and becomes negative and the flow becomes stable again when $\alpha \gtrsim 4 \times 10^{3}$. In the present analysis, we assume that the kinematic eddy viscosity in the vegetated zone is represented by (10) since the typical length scale of vegetation is not significantly smaller than the flow depth. When the vegetation density reaches a sufficiently large density, this assumption may no longer be valid. However, it is natural that the flow becomes stable with increasing $\alpha$ since the large-scale horizontal vortices are damped by strong retardation effects when $\alpha$ is sufficiently large. Therefore, the contours of $\Omega$ in the range of large $\alpha$ in Fig. 5 are at least qualitatively correct.

The dependence of $\Omega$ on the Froude number $F$ is studied in Fig. 6. It is found from the figure that the flow is unstable in the range of small and moderate $F$. As $F$ increases, the instability weakens and a stable region is observed in the range $F \approx 2.3-2.6$. When $F \gtrsim 2.6$, the flow is again

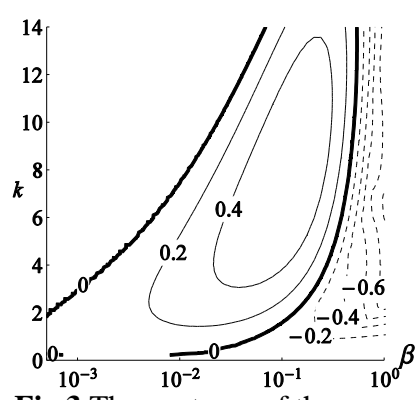

Fig.3 The contours of the growth rate $\Omega$ in the $\beta-k$ plane for the case $\epsilon=6 \times 10^{-4}, \alpha=10$, $B_{v}=0.55, F=0.5$.

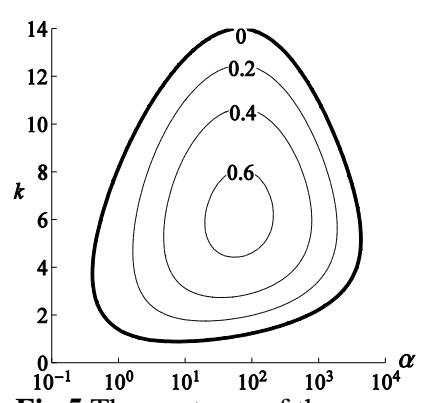

Fig.5 The contours of the growth rate $\Omega$ in the $\alpha-k$ plane for the case $\beta=0.05$, $\epsilon=6 \times 10^{-4}$, $B_{v}=0.55, F=0.5$.

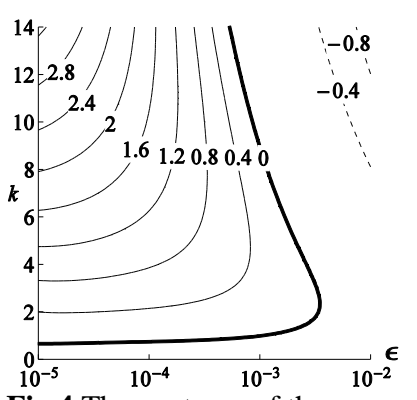

Fig.4 The contours of the growth rate $\Omega$ in the $\epsilon-k$ plane for the case $\beta=0.05, \alpha=10$, $B_{v}=0.55, F=0.5$.

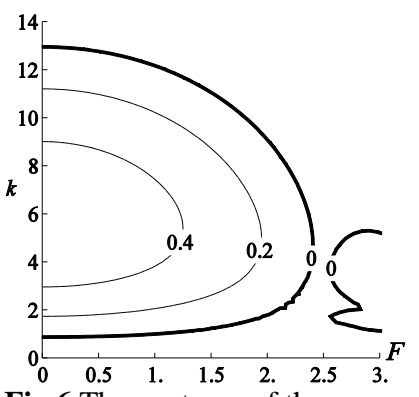

Fig.6 The contours of the growth rate $\Omega$ in the $F-k$ plane for the case $\beta=0.05$,

$\epsilon=6 \times 10^{-4}, \alpha=10$, $B_{v}=0.55$. 


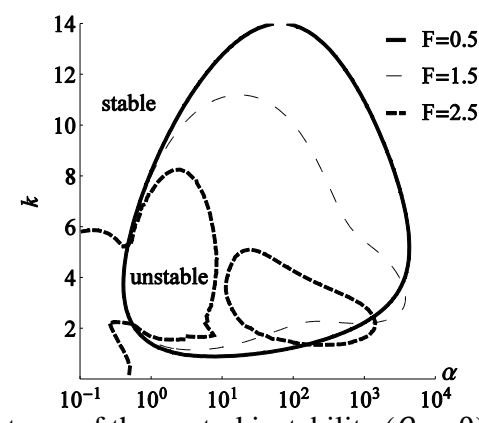

Fig.7 The contours of the neutral instability $(\Omega=0)$ in the $\alpha-k$ plane for the case $\beta=0.05, \epsilon=6 \times 10^{-4}, B_{v}=0.55$ and multiple values of $F$.

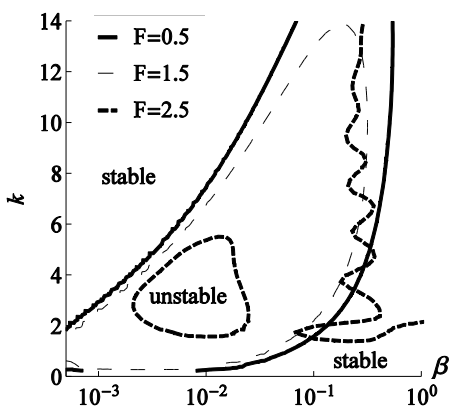

Fig.8 The contours of the neutral instability $(\Omega=0)$ in the $\beta-k$ plane for the case $\epsilon=6 \times 10^{-4}, \alpha=10, B_{v}=0.55$ and multiple values of $F$.

found to be unstable. It has been empirically known that rapid flow plays stabilizing effects for lateral velocity gradients (e.g. Ref. 2), what is theoretically explained in this analysis. On the other side, as pointed by Kolyshkin and Ghidaoui, ${ }^{5)}$ the rapid flow becomes unstable to gravity waves if $F$ is sufficiently large. Therefore, although in Fig. 6 the flow is found to be stable to the lateral velocity gradients when $F \gtrsim 2.3$, it is unstable to gravity when $F \gtrsim 2.6$.

Fig. 7 depicts the neutral instability curves $(\Omega=0)$ in the $\alpha-k$ plane for multiple values of $F$. While the flow is stable when $\alpha \lesssim 0.5$ for $F=0.5$ and $F=1.5$, it becomes unstable to gravity in this range when $F=2.5$. As $F$ increases from 0.5 to 2.5 , the unstable region for $\alpha \gtrsim 0.5$ diminishes because the gravity effects weaken the instability due to the transverse mixing. The combined effect of rapid flow and lateral mixing stabilizes the flow in the range of $\alpha \approx 10^{1}$ for $F=2.5$.

Fig. 8 depicts neutral instability curves $(\Omega=0)$ in the $\beta-k$ plane for multiple values of $F$. For $F=2.5$, the instability due to the drag difference is reduced and limited to the range of $\beta \cong 10^{-2}$. Additionally, for $F=2.5$ and in the range of $\beta \gtrsim 10^{-1}$, the channel is sufficiently wide to allocate gravity instabilities which develop independently of the shear layer and do not possess a preferential wavenumber for maximum instability.

\section{(2) Application to experimental data}

Results from the present analysis were validated using data from the laboratory experiments of Ikeda et al., ${ }^{12)}$ Tsujimoto $^{13)}$ and White and Nepf. ${ }^{7)}$ In their experiments, vortices were generated by an array of regularly spaced cylinders installed on one side of channels, which is the same setup assumed in the present analysis. Major hydraulic parameters of the experiments are listed in Table 1.

Fig. 9 shows the neutral instability curve $(\Omega=$ $0)$ in the $\alpha-\beta$ plane for the case $\epsilon=10^{-3.0}$, $B_{v}=0.43$ and $F=1.0$. Although this curve represents a condition which is less favorable to the occurrence of unstable flow when compared to the experiments, it predicts unstable flow for most experimental runs, with exception of runs VII-XI, which have large values of $\alpha$. Because the range of large $\alpha$ may be stabilized at $F=1.0$, runs VII-XI

Table 1 Hydraulic variables from the experiments of Ikeda $e t$ $a^{12)}{ }^{12)}$ (runs 1-5, $B_{v}=0.59$ ), Tsujimoto ${ }^{13)}$ (runs IW1-3,

$B_{v}=0.43$ ) and White and $\mathrm{Nepf}^{7)}$ (runs I-XI, $B_{v}=0.50$ ).

\begin{tabular}{|c|c|r|r|c|c|c|c|r|r|r|r|}
\hline Run & $\beta$ & $\begin{array}{c}\epsilon \\
\left(\times 10^{-4}\right)\end{array}$ & $\alpha$ & $F$ & St & Run & $\beta$ & $\begin{array}{c}\epsilon \\
\left(\times 10^{-4}\right)\end{array}$ & $\alpha$ & $F$ & St \\
\hline \hline $\mathbf{1}$ & .061 & 5.2 & 9.9 & .51 & .040 & III & .023 & 7.3 & 160 & .21 & .042 \\
\hline $\mathbf{2}$ & .061 & 5.2 & 9.9 & .77 & .039 & IV & .064 & 4.0 & 180 & .22 & .039 \\
\hline $\mathbf{3}$ & .079 & 4.5 & 6.9 & .74 & .039 & $\mathbf{V}$ & .061 & 2.8 & 160 & .05 & .040 \\
\hline $\mathbf{4}$ & .164 & 3.0 & 3.7 & .62 & .037 & VI & .043 & 2.9 & 240 & .16 & .040 \\
\hline $\mathbf{5}$ & .038 & 6.9 & 16 & .48 & .041 & VII & .055 & 3.7 & 1800 & .21 & .036 \\
\hline IW1 & .024 & 4.8 & 68 & .69 & .042 & VIII & .130 & 4.3 & 870 & .08 & .034 \\
\hline IW2 & .030 & 5.9 & 51 & .78 & .042 & IX & .089 & 4.9 & 920 & .11 & .035 \\
\hline IW3 & .025 & 5.4 & 64 & .93 & .042 & X & .066 & 5.2 & 1100 & .34 & .035 \\
\hline I & .058 & 4.0 & 64 & .22 & .040 & XI & .032 & 8.7 & 3000 & .19 & .037 \\
\hline II & .023 & 4.7 & 160 & .21 & .042 & & & & & & \\
\hline
\end{tabular}

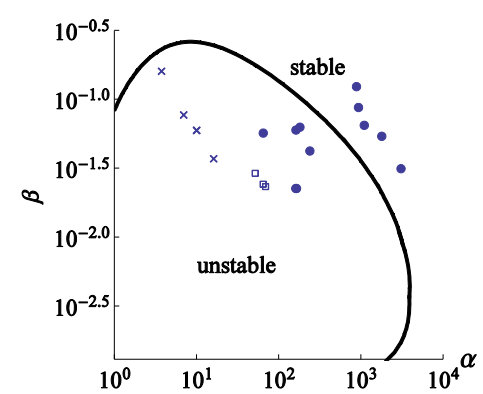

Fig.9 The contour of the neutral instability $(\Omega=0)$ in the $\beta$ - $\alpha$ plane for the case $\epsilon=10^{-3.0}, B_{v}=0.43, F=1.0$; experimental data: $\times$ Ikeda et al., ${ }^{12)} \square$ Tsujimoto, ${ }^{13)} \bullet$ White and Nepf. ${ }^{7}$

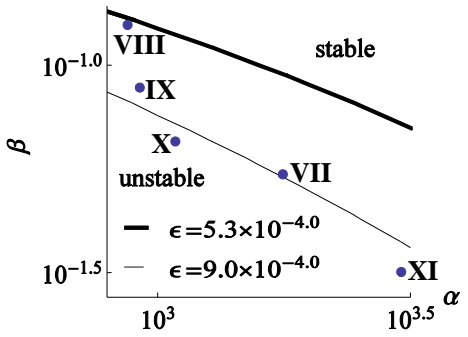

Fig.10 The contours of the neutral instability $(\Omega=0)$ in the $\beta-\alpha$ plane for the case $B_{v}=0.50, F=0.35$ and multiple values of $\epsilon$. Experimental data: $\bullet$ White and Nepf, ${ }^{7)}$ runs VII-XI. 


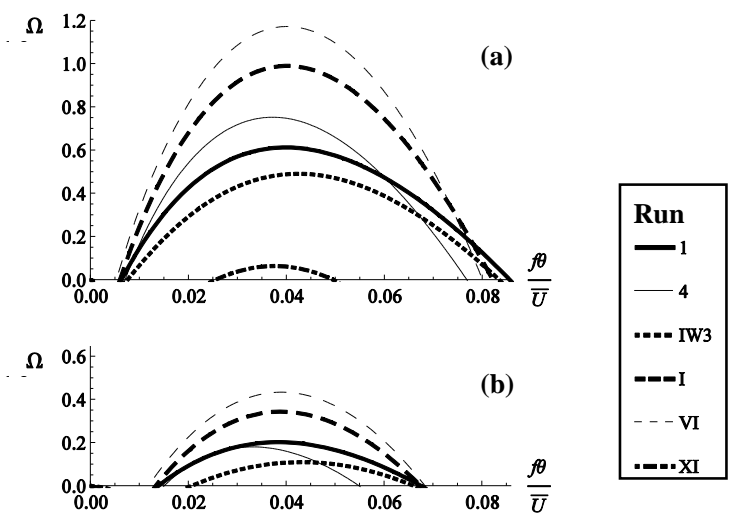

Fig.11 Variation of $\Omega$ with $f \theta_{0} / \bar{U}$ for selected experimental runs of Refs.7, 12, 13: (a) kinematic eddy viscosity from equation (10); (b) increased kinematic eddy viscosity, no positive $\Omega$ for run XI.

are restudied in Fig. 10. The flow is confirmed to be unstable in the case $\epsilon=5.3 \times 10^{-4.0}$ for runs VII-X, and in the case $\epsilon=9.0 \times 10^{-4.0}$ for run XI.

The Strouhal number $\mathrm{St}=f_{m} \theta_{0} / \bar{U}$ for the maximum growth rate in a shear layer takes the nearly constant value of 0.032 for any hyperbolic tangent base flow profile, ${ }^{14)}$ where $f_{m}$ is the natural frequency for maximum instability, $\theta_{0}$ is the momentum thickness of the base flow and $\bar{U}$ is the average of the far field velocities $(\bar{U}=(1+\phi) / 2)$. Table 1 presents the Strouhal numbers of the most amplified waves determined herein. In addition, curves of the variation of $\Omega$ with $f \theta_{0} / \bar{U}$ determined herein are presented in Fig. 11(a). The values of St in Table 1 and the values of $f \theta_{0} / \bar{U}$ for the maximum $\Omega$ in Fig. 11(a) are slightly larger than 0.032. Based on the fact that the development of the perturbations reduce $\mathrm{St}$, this overestimation may be because we employed a base flow velocity which is set completely free of the lateral motions due to the shear layer, while the hyperbolic-tangent velocity profile from which St was determined as 0.032 may have effects of such lateral motions.

In case the increased kinematic eddy viscosity $\tilde{v}_{T}=0.2 \widetilde{U}_{f} \widetilde{H}_{\infty}$ had been employed, the growth rate $\Omega$ would have been underestimated, as depicted in Fig. 11(b).

\section{CONCLUSIONS}

A temporal linear stability analysis of open-channel flows with lateral vegetation is performed. Small perturbations are imposed to a base flow which is set as the flow unaffected by the large-scale vortices which develop around the edge of the vegetated zone. Because the rigid-lid assumption was not employed in the present analysis, the growth rate of perturbations could also be studied for Froude numbers which are not close to zero. In addition, the viscous flow formulation with the use of a theoretical sub-depth kinematic eddy viscosity unaffected by lateral large-scale motions permitted a consistent estimation of the growth rate of perturbations.

Our results indicate that, while the base state flow field is unstable in the range of typical, moderate values of the hydraulic parameters, stability is retained in the range of sufficiently small and large vegetation densities, large bed shear effect, large sub-depth eddy viscosity effect, and moderate Froude numbers where the flow is stable to both the transverse mixing and the gravity. The application of the model into experimental data supports the theory.

\section{REFERENCES}

1) Tamai, N., Asaeda, T. and Ikeda, H.: Study on generation of periodical large surface eddies in a composite channel flow, Water Resour. Res., Vol.22, No.7, pp.1129-38, 1986.

2) Chu, V.H., Wu, J.-H. and Khayat, R.E.: Stability of transverse shear flows in shallow open channels, J. Hydraul. Eng.-ASCE, Vol.117, pp.1370-88, 1991.

3) Uijttewaal, W.S.J. and Booij, R.: Effects of shallowness on the development of free-surface mixing layers, Phys. Fluids, Vol.12, pp.4392-02, 2000.

4) Prooijen, B.C. and Uijttewaal, W.S.J.: A linear approach for the evolution of coherent structures in shallow mixing layers, Phys. Fluids, Vol.14, pp.4105-14, 2002.

5) Kolyshkin, A.A. and Ghidaoui, M.S.: Gravitational and shear instabilities in compound and composite channels, J. Hydraul. Eng.-ASCE, Vol.128, pp.1076-86, 2002.

6) Ikeda, S., Ohta, K. and Hasegawa, H.: Instability-induced horizontal vortices in shallow open-channel flows with an inflection point in skewed velocity profile, J. of Hydroscience and Hydraulic Engineering, Vol.12, No.2, pp.69-84, 1994.

7) White, B. and Nepf, H.: Shear instability and coherent structures in shallow flow adjacent to a porous layer, J. Fluid Mech., No.593, pp.1-32, 2007.

8) Ghidaoui, M.S. and Kolyshkin, A.A.: Linear stability analysis of lateral motions in compound open channel, J. Hydraul. Eng.-ASCE, Vol.125, pp.871-880, 1999.

9) Lima, A.C. and Izumi, N.: Fully developed stage of perturbations in parallel transverse shear flows, J. of Japan Society of Civil Engineers, Ser. B1 (Hydraulic Engineering), Vol.67, No.4, pp.I73-I78, 2013.

10) Fischer, H.B., List, E.J., Koh, R.C.J., Imberger, J. and Brooks, N.H.: Mixing in inland and coastal waters, Academic, 1979.

11) Chen D. and Jirka J.H.: Absolute and convective instabilities of plane turbulent wakes in a shallow water layer, J. Fluid Mech., No.338, pp.157-71, 1997.

12) Ikeda, S., Izumi, N. and Ito, R.: Effects of pile dikes of flow retardation and sediment transport, J. Hydraul. Eng.-ASCE, Vol.117, pp.1459-78, 1991.

13) Tsujimoto, T.: Open channel flow with bank vegetation, KHL Communication, No.2, pp.41-54, 1991 (in Japanese).

14) Ho, C.M. and Huerre, P.: Perturbed free shear layers, Annu. Rev. Fluid Mech., No.16, pp.365-24, 1984.

(Received September 30, 2013) 